\title{
Social Robot Encouraging Two Strangers to Talk with Each Other for Their Relationships
}

\author{
Naoki Ise \\ Master's Program in Intelligent Interaction Technologies \\ University of Tsukuba \\ Tsukuba, Japan \\ s2020705@s.tsukuba.ac.jp
}

\author{
Takamasa Iio \\ Faculty of Engineering, Information and Systems \\ University of Tsukuba \\ Tsukuba, Japan \\ iio@iit.tsukuba.ac.jp
}

\begin{abstract}
We investigated a hypothesis that a conversation between two strangers would be encouraged and they would improve each other's likability if a robot induced them to have a small talk in advance. In our experiment a participant remained in a waiting space with an experimental cooperator for five minutes, and then they did a collaborative task together. The experiment had two conditions: One was the with-intervention condition in which a robot asked them to do a small talk when they remained in the waiting space, another was the without-intervention condition in which there was no robot in the waiting space. The results showed that the number of participant's utterances in the collaborative task had no significant difference $(t=-0.676, p=0.511, d=-0.350)$, and likability rating of the cooperator by the participant also had no significant difference $(\mathrm{t}=-0.781, \mathrm{p}=0.449, \mathrm{~d}=0.404)$. These results did not support the hypothesis. It suggested that it was difficult to affect a relationship between two strangers by a robot prompting them to speak simply.
\end{abstract}

\section{CCS CONCEPTS}

- Human-centered computing --- Scenario-based design; Empilical studies in interaction design; • Applied computing --- Sociology

KEYWORDS: Human-robot interaction; Social robotics; Social relationship; Assisting conversation

\section{ACM Reference format:}

Naoki Ise, Takamasa Iio. 2021. Social Robot Encouraging Two Strangers to Talk with Each Other for Their Relationships. In Proceeding of 2021 ACM/IEEE International Conference on Human-Robot Interaction (HRI'21 Companion), March 8-11, 2021, Boulder, CO, USA. ACM, New York, NY, USA, 4 pages. https://doi.org/10.1145/3434074.3447147

\section{Introduction}

The number of young people who are not good at face-to-face communication has been increasing in recent years [1,2]. Reduced

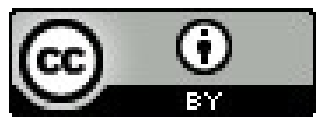

This work is licensed under a Creative Commons Attribution International 4.0 License.

HRI '21 Companion, March 8-11, 2021, Boulder, CO, USA.

(C) 2021 Copyright held by the owner/author(s).

ACM ISBN 978-1-4503-8290-8/21/03. https://doi.org/10.1145/3434074.3447129 face-to-face communication can cause serious problems; for example, chronic loneliness and a reduction in their social capital [3]. Chronic loneliness has been reported to be associated with increased risk for early mortality [4]. Reduced social capital can make communities unstable in the aspect of poverty, unemployment, crime and so on [5,6]. To increase the chance of face-to-face communication would be important for our society.

To address the problem of reduced face-to-face communication, we believe social robots could be used. Social robots that work in the same physical space as humans, such as nursing homes for the elderly [7], schools [8], and shopping malls [9], can become an interface between strangers and encourage them to communicate. For example, in a school cafeteria, a robot might take a child who feels isolated to another child who feels isolated as well, and let them have lunch together. Although these services may seem like meddling, they would be a good method to start a little conversation with strangers.

However, encouraging face-to-face communication through a social robot have not been studied so much. Many studies have focused on the communication between a robot and a person. The basic knowledge regarding how robot intervention affects face-toface communication between people is unclear for now.

Therefore, as a first step, we investigate the following naive hypothesis of the effects of robot intervention: "A conversation between two strangers would be encouraged and they would improve each other's likability if a robot induced them to have a small talk in advance." The hypothesis is based on the following simple idea: After a robot induced them to talk to each other, they would reduce the psychological burden of speaking something in the subsequent conversation. Furthermore, it would increase their liking for each other in terms of mere-exposure effect [10]. This paper reports an experiment to verify that hypothesis and the results.

\section{Method}

\subsection{Participants and cooperators}

Fifteen participants and two cooperators were participated. The participants were first-third grade university students. The average age was $18.87(\mathrm{SD}=0.718)$, and they were all males. The two cooperators were fourth grade students of the same university. Their ages were 22 , and they were also males. The reason for limiting gender and age of the participants and the 
cooperators was that it was difficult to investigate all combinations (e.g. female-female, male-male and female-male, younger-elder, elder-younger and same age). In preliminary tests, we had observed that two females tended to have more utterances in a conversation than two males, and elder people were likely to take an initiative in the conversation more than younger people. In this paper, we focused on the combination of a younger male participant and an elder male cooperator because we assumed that the younger person would not talk so much in the combination.

Furthermore, we measured the social skills of the participants using the Kiss18 (Kikuchi's social skills scale) [11]. Social skills are defined as a series of cognitive, emotional, and behavioral processes used in interpersonal situations [12]. Thus, they are closely related to communications between strangers. For example, a participant with high social skills may talk to the cooperator easily. That is why, we needed to pay attention to the social skills. Kiss-18 has been applied in many studies to measure social skills of Japanese people [13]. The average score was 58.27 ( $\mathrm{SD}=8.32$ ).

\subsection{Design}

The experiment had the follow two conditions:

- With-intervention condition: A robot induced two people (a participant and a cooperator) to have a small talk while they are waiting for task preparation.

- Without-intervention condition: There was no robot in the waiting space so that the two people were not intervened by anyone while they are waiting for task preparation.

The experiment followed the between-participants design. The participants were assigned to either of the conditions so that the average social skill score was almost equal between the conditions. The average social skill score of the with-intervention condition was $59.29(\mathrm{SD}=9.28)$, and that of the without-intervention condition was $57.38(\mathrm{SD}=7.26)$.

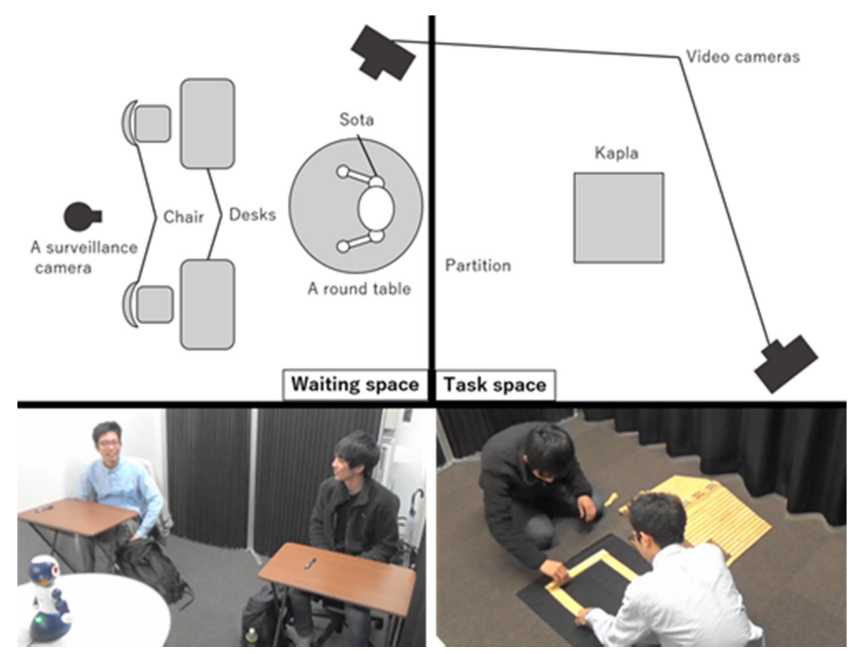

Figure 1: Outline drawing of the experimental environment.

\subsection{Environment and apparatus}

The environment diagram and the pictures are shown in Figure 1. It was divided into two spaces: The waiting space and the task space. The waiting space was a space where a participant and a cooperator stayed at first and filled a questionnaire after the task.

The task space was the space where the two people performed a collaborative task. These spaces were separated by a partition so that they could not see the other spaces. We put a video camera to record participant's behavior.

We used 'Sota' robot, developed by Vstone Co., Ltd. This robot is a table top type robot approximately $30 \mathrm{~cm}$ in height, and it has 3 degrees of freedom in the head, 2 degrees of freedom in both arms, and 1 degree of freedom in the body. The robot acted according to a predetermined conversation sequence. Several utterances and gestures for participants' responses were prepared, and an operator selected the appropriate one by remote control.

We used toy-blocks called 'Kapla' for the collaboration task. Kapla can be stacked in various ways and played with in creative ways. They are often used in psychological experiments as a tool to get people to collaborate [14]. The number of the blocks was 1500, which was considered to be enough for novices to build something for $20 \mathrm{~min}$.

\subsection{Procedure}

Before the experiment, we explained the purpose and overview of the experiment to the participants several days in advance and obtained informed consent from them. At that time, the participants filled in a questionnaire of social skills (Kiss-18).

The procedure was separated into three phases: Waiting phase, task phase, and filling-in-the-questionnaire phase.

\subsubsection{Waiting phase}

A cooperator sat on a chair in the waiting space, watching his own smartphone. When a participant came in the experimental room, an experimenter took him to another chair in the waiting space and asked him to sit down. Then, the experimenter instructed the participant and the cooperator as follows: "I'm preparing for this experiment. Please wait a while. Take it easy." Then, the experimenter left. The participant and the cooperator stayed there for five minutes. If the participant was a part of the withintervention condition, an operator ran the program of the robot's intervention. A part of the content of the talk is shown in Table 1. Otherwise, the participant and the cooperator just waited as they pleased.

Table 1: A part of the content of the talk.

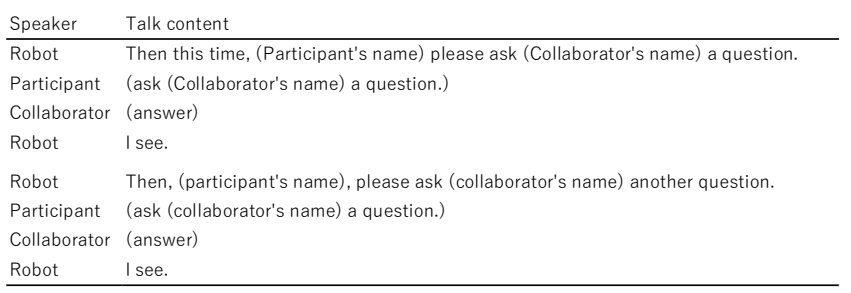

In the with-intervention condition, a cooperator watched his own smartphone until a robot started to talk. After the robot started talking, the cooperator talked with the robot and the 
participant according to the scenario we had prepared. In the without-intervention condition, a cooperator watched his own smartphone until an experimenter came to take him and the participant to the task space.

The reason for letting the cooperator watch his smartphone was to reproduce a situation of common waiting spaces. In general, people in waiting spaces such as hospitals, hotel lobbies, and shopping malls do what they like; for example, they watch their smartphones, listen to music, and read books. However, in a preliminary experiment, we observed that some participants had a conversation with a cooperator. We considered that this might be because the participants were not sure of the meaning of taking it easy. Therefore, to make sure that participants were aware that they could watch their smartphones while they were waiting, we had the cooperator watch his smartphone.

\subsubsection{Task phase}

After five minutes, the experimenter explained to the participant and the cooperator that today's task was a two-person and took into the task space. The experimenter instructed, "Please create something you like, using this building blocks called Kapla. The time limit is 20 minutes." Then, the experimenter set a timer and left. The participant and the cooperator performed the task together for $20 \mathrm{~min}$.

The cooperators performed the task with the participants according to the following rules:

- A cooperator does not proactively try to start a conversation with a participant.

- When a participant asks the cooperator a question, the cooperator can answer the question.

- A cooperator can perform back-channeling with respect to the participant's utterances within reason.

The reason for these rules was to ensure that the cooperators behaved in a consistent manner. Since the cooperator's behavior clearly influences the amount of participant's utterances and the impression of the cooperator, it was necessary to control the cooperator's behavior to minimize its influence on the results.

2.4.3 Filling-in-a-questionnaire phase: After 20 minutes, the experimenter took the participant and the cooperator into the waiting space and had them sit down on their chairs. Then, the experimenter asked the participant and the cooperator to fill in a questionnaire. (The cooperator also answered the questionnaire, but the results were not used.) After filling the questionnaire, the experimenter briefed the participant.

The above procedure was approved by the ethical committee of our university (This is anonymized. If this paper is accepted, we'll write it).

\subsection{Operator role}

An operator controlled the robot remotely. The operator run the intervention program of the robot after an experimenter left the waiting space in the waiting phase. The intervention program had a sequential structure, in which sentences the robot spoke were regarded as nodes. The operator executed each node step by step, observing reactions of the participant and the cooperator through the camera installed in the waiting space.

Indeed, in future, a robot will intervene using various sensing capabilities such as speech recognition and face recognition, but because the aim of this experiment was to investigate the influence of robot's intervention, we controlled the robot using an operator so that the experiment was nor hindered by speech recognition errors.

\subsection{Measurements}

We measured the following aspects of each participant:

- Total speaking time of the participants in the task phase.

- $\quad$ Liking scale [15]

To measure the total speaking time of the participants in the task phase, we made a transcript of the utterances of the participants in the task phase and annotated the beginning time and the end time of the utterances. Here, we divided each utterance by a no-utterance interval of more than $300 \mathrm{msec}$.

Liking scale is a part of Love-liking scale developed by Rubin [16]. In fact, we used the Japanese version of the Liking scale developed by Fujiwara et al. [15]. The Liking scale can measure how people feel regarding the pleasant features of a target person. Although Rubin's original scale has 13 items, we removed the first, sixth, seventh, and eleventh items from the scale because Fujiwara et al. pointed out that these items should be removed in terms of validity. Thus, the range of the scores was from 9 to 81 .

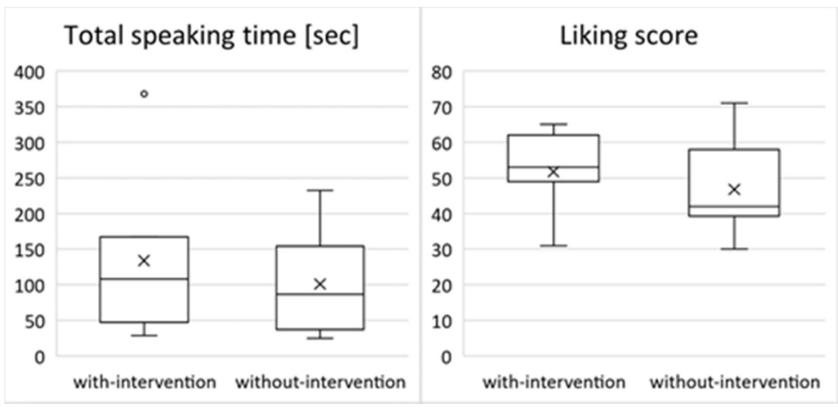

Figure 2: The average Total speaking time (left) and Liking score (right) of each condition.

We compared each measurement between the two conditions by a t-test. The significance level $\alpha$ was set at 0.05 .

\section{Results}

Figure 2 shows the results of the total speaking time and the liking scores. We explain the detail of them below.

\subsection{Total speaking time}

The average total speaking time of the with-intervention condition was $134 \mathrm{sec}(\mathrm{SD}=106 \mathrm{sec})$, and that of the without-intervention condition was $101 \mathrm{sec}(\mathrm{SD}=67 \mathrm{sec})$. These results are shown in 
Figure 2(left). The t-test showed no significant difference between the conditions $(t=-0.676, p=0.511, d=-0.350)$.

\subsection{Liking score}

The average liking score of the with-intervention condition was $51.71(\mathrm{SD}=10.21)$, and that of the without-intervention condition was 46.75 ( $\mathrm{SD}=12.40)$. These results are shown in Figure 2(right). The t-test showed no significant difference between the conditions $(\mathrm{t}=0.781, \mathrm{p}=0.449, \mathrm{~d}=0.404)$.

\section{Discussion}

\subsection{Implications}

The results did not support our hypothesis that when a robot forced a conversation between strangers, their utterances would increase in the subsequent conversation and they would start liking each other. Regarding the total speaking time, there was no significant difference between the average total speaking time of the with-intervention condition and that of the withoutintervention condition. We cannot conclude that the robot's intervention affected the amount of participant's utterances. Regarding liking scores, there were no significant differences between the average liking score of the with-intervention condition and that of the without-intervention condition. We cannot conclude that robot's intervention influenced the participant's impressions for the cooperator.

The reason for little effect of robot's intervention may be due to short intervention times and one-time encounters. The intervention time in the current experiment was approximately 5 minutes, which may not have been enough time to alleviate the participants' psychological barriers to their collaborators. However, in a real environment such as a school cafeteria or a lounge of a nursing care home, the robot cannot be expected to intervene for a long time. Therefore, it is necessary to think of ways to encourage a conversation between two people, even if it is only for 5 minutes. In addition to the short intervention time, the robot only intervened once in the experiment. The mere exposure effect, on which our hypothesis is based, assumes repeated exposure to things. In that sense, a single intervention may not have influenced participants' speech time or impressions. In order to examine the impact of the number of interventions in the future, we plan to incorporate encounters over several days into the experimental design. We speculate that the effect of robot intervention will be apparent when we experiment for several days.

\subsection{Limitations}

The participants of this experiment were only males and university students. The cooperators were also males and university students. The participants were younger than the cooperators. The cooperators did not proactively speak to the participants. Therefore, the findings and discussions in this study would be available only when male university students interact with an older male university student with a passive attitude.

\section{ACKNOWLEDGMENTS}

This study was funded by JST PRESTO Grant Number JPMJPR1851, Japan.

\section{REFERENCES}

[1] Turkle, S. (2017). Alone together: Why we expect more from technology and less from each other. Hachette UK.

[2] Cacioppo, J. T., and Patrick, W. (2008). Loneliness: Human nature and the need for social connection. WW Norton \& Company.

[3] Putnam, R. D. (2000). Bowling alone: The collapse and revival of American community. Simon and Schuster

[4] Holt-Lunstad, J., Smith, T. B., Baker, M., Harris, T., and Stephenson, D. (2015) Loneliness and social isolation as risk factors for mortality: a meta-analytic review. Perspectives on psychological science, 10(2), 227-237.

[5] Grootaert, C., Narayan, D., Jones, V. N., and Woolcock, M. (2004). Measuring social capital: An integrated questionnaire. The World Bank.

[6] Temple, J. (2002). Growth effects of education and social capital in the OECD countries. Historical Social Research/Historische Sozialforschung, 5-46.

[7] Iio, T., Yoshikawa, Y., Chiba, M., Asami, T., Isoda, Y., and Ishiguro, H. (2020). Twin-Robot Dialogue System with Robustness against Speech Recognition Failure in Human-Robot Dialogue with Elderly People, Appl. Sci. 10(4)

[8] Kanda, T., Sato, R., Saiwaki, N., and Ishiguro, H. (2007). A twomonth field trial in an elementary school for long-term human-robot interaction. IEEE Transactions on robotics, 23(5), 962-971.

[9] Kanda, T., Shiomi, M., Miyashita, Z., Ishiguro, H., and Hagita, N. (2009, March). An affective guide robot in a shopping mall. In Proceedings of the 4th ACM/IEEE international conference on Human robot interaction (pp. 173-180).

[10] Zajonc, R. B. (2001). Mere exposure: A gateway to the subliminal. Current directions in psychological science, 10(6), 224-228.

[11] Kikuchi, A., and Horike, K. (1994). Psychology of social skill. Kawashima Shoten.[in Japanese].

[12] Hargie, O. (Ed.). (2006). The handbook of communication skills.

[13] Katagami, D., Ohmura, H., and Nitta, K. (2010, July). Investigation of social adaptive skills by cross-cultural simulation game and KiSS-18. In International Conference on Fuzzy Systems (pp. 1-6). IEEE.

[14] Stivers, T., and Sidnell, J. (2016). Proposals for activity collaboration. Research on Language and Social Interaction, 49(2), 148-166.

[15] Fujihara, T., Kurokawa, M., and Akizuki, S. (1983). Factor analytical study of Rubin's love and liking scales. Memoirs of Faculty of Integrated, Arts and Science III, Studies in Information and Behavioral Sciences, 7, 39-46.

[16] Rubin, Z. (1970). Measurement of romantic love. Journal of personality and social psychology, 16(2), 265. 